\title{
Influencing factors of transient elastography in detecting liver stiffness
}

\author{
RONG SHAN ${ }^{1,2}$, HONG YIN $^{3}$, WENJUAN YANG ${ }^{4}$, JIANZHI LI $^{2}$, MEIFANG ZHANG $^{2}$, \\ MIN ZHAO $^{2}$, JIANG SHAO ${ }^{2}$ and AIGUANG WANG ${ }^{5}$ \\ ${ }^{1}$ Department of Ultrasound, Shandong University, Jinan, Shandong $250014 ;{ }^{2}$ Department of Ultrasound, Jinan Infectious
Disease Hospital, Jinan, Shandong 250021; ${ }^{3}$ Department of Ultrasound, Jinan Maternity and Child Care Hospital,
Jinan, Shandong 250000; ${ }^{4}$ Department of Emergency, Jinan Center Hospital, Jinan, Shandong 250001;
${ }^{5}$ Department of Oncology, Qianfoshan Hospital Affiliated to Shandong University, Jinan, Shandong 250014, P.R. China
}

Received April 28, 2015; Accepted May 20, 2015

DOI: $10.3892 /$ etm.2016.3617

\begin{abstract}
Liver stiffness, which correlates well with liver fibrosis stage, can be measured noninvasively by transient elastography, also known as Fibroscan. The present study aimed to determine the independent factors influencing Fibroscan detection by multiple regression analysis. A total of 181 patients who required liver biopsy were enrolled. Liver stiffness measurement (LSM) was detected by Fibroscan on the day of liver biopsy, while clinical information and routine biochemical examination results were also collected. Correlation was analyzed by Spearman's correlation, and multiple regression analysis was performed to analyze the independent influencing factors. The results demonstrated that platelet (PLT) levels, serum albumin (ALB), prothrombin activity (PTA) and body mass index (BMI) were independent predictors of liver stiffness. The contribution of these four predictors to the regression equation was in the following descending order: PLT (negative correlation) > ALB (negative correlation) $>$ PTA (negative correlation) $>$ BMI (positive correlation). In conclusion, the parameters of PLT, ALB, PTA and BMI are independent predicting factors affecting Fibroscan detection. Therefore, the diagnosis and evaluation of liver fibrosis should comprehensively consider the results of Fibroscan, and clinical and laboratory examinations.
\end{abstract}

Correspondence to: Dr Aiguang Wang, Department of Oncology, Qianfoshan Hospital Affiliated to Shandong University, 16766 Jingshi Road, Jinan, Shandong 250014, P.R. China

E-mail: sr11911@126.com

Key words: Fibroscan, liver stiffness, multiple regression analysis, independent predictor

\section{Introduction}

Hepatitis B virus (HBV) infection is prevalent around the world, and is one of the most severe infectious diseases in China. An epidemiological survey of hepatitis B in 2006 demonstrated that $7.18 \%$ of the Chinese population aged 1-59 years carried the hepatitis B surface antigen (HBsAg) (1). Accordingly, 93 million individuals in China suffer from chronic HBV infection (2). HBV infection can cause a series of pathological changes in the liver, including mild to severe liver fibrosis and severe hepatitis. These lesions can progress to cirrhosis, liver failure and end-stage liver disease (3).

Liver acute or chronic inflammation is frequently accompanied by the formation and development of liver fibrosis (4-6). The clinical manifestations of liver fibrosis and cirrhosis are different. Mild liver fibrosis can be completely asymptomatic, while severe liver fibrosis can be manifested as hepatic failure. Similarly, early stage cirrhosis has no evident symptoms or no specific clinical symptoms; however, advanced liver cirrhosis has multisystem manifestations of hepatic decomposition, including hemorrhage, infection, hepatic encephalopathy and carcinogenesis $(3,7)$. The 5-year mortality rate of early cirrhosis is $<20 \%$ and the 10 -year survival rate is $\sim 70 \%$; by contrast, the 5 -year survival rate of decompensated cirrhosis is $<50 \%$ (8). Thus, the timely diagnosis of liver fibrosis has an important value in treatment and prognostic assessment of chronic liver disease.

Transient elastography, also known as Fibroscan, is a new, noninvasive, indolent, fast and objectively quantitative method for evaluating liver fibrosis by measuring the liver stiffness (9). Castéra et al (9) and Colletta et al (10) have reported that detecting liver fibrosis by Fibroscan resulted in 84-95\% of patients not requiring liver biopsy in 2005. Fibroscan has previously been used to evaluate the degree of liver fibrosis in patients with hepatitis B $(11)$, hepatitis C $(12,13)$ cirrhosis (14), alcoholic liver disease $(15,16)$ and autoimmune liver diseases (17). It is also used in antiviral therapy efficacy evaluation $(18,19)$.

The aim of the present study was to investigate the independent predictors affecting Fibroscan detection of liver fibrosis, and the method of multiple regression analysis was used to determine these predictors. 
Table I. Clinical features of patients $(n=181)$.

\begin{tabular}{|c|c|c|}
\hline Index & Value & Normal range \\
\hline Male to female ratio $[\mathrm{n}(\%)]$ & $128: 53(70.7 \%: 29.3 \%)$ & \\
\hline Age (years) & $38.7 \pm 10.8(17-72)$ & \\
\hline BMI $\left(\mathrm{kg} / \mathrm{m}^{2}\right)$ & $24.3 \pm 3.3(16.5-34.4)$ & $18.5-24.99$ \\
\hline Liver stiffness measurement $(\mathrm{kPa})$ & $11.9 \pm 11.9(2.5-75.0)$ & \\
\hline \multicolumn{3}{|l|}{ Pathological results [n (\%)] } \\
\hline Hepatitis B & $154(85.1)$ & \\
\hline Hepatitis C & $2(1.1)$ & \\
\hline Autoimmune hepatitis & $18(9.9)$ & \\
\hline Fatty liver & $3(1.7)$ & \\
\hline Non-specific liver damage & $4(2.2)$ & \\
\hline $\operatorname{PLT}\left(\times 10^{3} / \mathrm{mm}^{3}\right)$ & $187.4 \pm 67.6(54-388)$ & $100-300$ \\
\hline ALT (IU/1) & $65.9 \pm 69.9(7-548)$ & $0-40.0$ \\
\hline AST (IU/l) & $49.5 \pm 48.9(12-341)$ & $0-40.0$ \\
\hline GGT (IU/l) & $72.7 \pm 92.9(9-504)$ & $12.0-64.0$ \\
\hline $\operatorname{ALP}(\mathrm{IU} / 1)$ & $82.0 \pm 71.0(9-636)$ & $40.0-150.0$ \\
\hline $\operatorname{ALB}(\mathrm{g} / \mathrm{l})$ & $44.2 \pm 4.5(28.4-55.0)$ & $35.0-55.0$ \\
\hline GLOB (g/l) & $31.6 \pm 5.7(19.7-60.9)$ & $25.0-35.0$ \\
\hline $\mathrm{A} / \mathrm{G}$ ratio & $1.4 \pm 0.3(0.6-2.2)$ & $1.2-35.0$ \\
\hline BILT (mg/l) & $25.9 \pm 46.9(5.3-535.3)$ & $4.0-25.0$ \\
\hline DBIL (mg/l) & $9.9 \pm 27.7(1.7-301.8)$ & $1.0-10.0$ \\
\hline IBIL (mg/l) & $15.9 \pm 20.0(3.3-233.5)$ & $1.0-20.0$ \\
\hline CHEX (U/l) & $289.4 \pm 85.7(78-548)$ & $130.0-310.0$ \\
\hline PT (sec) & $13.9 \pm 1.4(11.3-19.2)$ & 8.8-13.8 \\
\hline PTA $(\%)$ & $90.3 \pm 16.4(51-145)$ & $70.0-130.0$ \\
\hline FIB (g/l) & $2.9 \pm 1.6(1.5-21.4)$ & $2.0-4.0$ \\
\hline
\end{tabular}

Numerical variable data are shown as the mean \pm standard deviation (range), unless otherwise stated. BMI, body-mass index; PLT, platelet; ALT, alanine aminotransferase; AST, aspartate aminotransferase; GGT, $\gamma$-glutamyl transpeptidase; ALP, alkaline phosphatase; ALB, serum albumin; GLOB, globulin; A/G, albumin/globulin; BILT, total bilirubin; DBIL, direct bilirubin; IBIL, indirect bilirubin; CHEX, cholinesterase; PT, prothrombin time; PTA, prothrombin activity; FIB, fibrinogen.

\section{Materials and methods}

Clinical data of subjects. A total of 181 cases were enrolled into the present study. These patients were diagnosed with chronic hepatitis, cirrhosis, fatty liver, autoimmune hepatitis or non-specific cholangitis by liver biopsy in the Outpatient and Inpatient Departments of the Jinan Infectious Disease Hospital (Jinan, China) during the period between June 2011 and June 2013. These cases included 128 male patients and 53 female patients, with ages ranging between 17 and 72 years and an mean age of $38.7 \pm 10.8$ years. Of the 181 included patients, 154 cases were HBsAg-positive. The present study was conducted in accordance with the Declaration of Helsinki, and with approval from the Ethics Committee of Qianfoshan Hospital, Shandong University (Jinan, China). Written informed consent was obtained from all participants.

Clinical information and biochemical examination. The analyzed clinical and biochemical indexes included the following: Gender, age, body-mass index (BMI), platelet (PLT), alanine aminotransferase (ALT), aspartate aminotransferase (AST), $\gamma$-glutamyl transpeptidase (GGT), alkaline phosphatase
(ALP), serum albumin (ALB), globulin (GLOB), ALB/GLOB ratio $(A / G)$, total bilirubin (BILT), direct bilirubin (DBIL), indirect bilirubin (IBIL), cholinesterase (CHEX), prothrombin time (PT), prothrombin activity (PTA) and fibrinogen (FIB). The BMI was calculated according to the following formula: BMI = body weight $(\mathrm{kg}) /$ height $^{2}\left(\mathrm{~m}^{2}\right)$. In addition, fasting blood was collected from all patients for biochemical analysis. PLT level was analyzed by an ADVIA 2120 hematology analyzer (Bayer Diagnostics, Tarrytown, NY, USA). The levels of ALT, AST, GGT, ALP, ALB, GLOB, A/G, BILT, DBIL, IBIL and CHEX were detected with an Abbott Architect c8000 biochemical analyzer (Abbott Diagnostics, Santa Clara, CA, USA). Finally, the PT, PTA and FIB levels were analyzed using a STAGO Compact automatic coagulation analyzer (Diagnostica Stago, Asnières-sur-Seine, France).

Fibroscan. Liver stiffness in the 181 patients was measured by Fibroscan (Echosens, Paris, France) prior to the liver biopsy on the same day. Briefly, the probe was place at the detection area from the anterior axillary line on the right to the 7th, 8th and 9th intercostal space in the midaxillary line for continuous detection. A total of 10 successful detections were performed 
Table II. Correlation analysis between liver stiffness measurement and various indexes.

\begin{tabular}{lcr}
\hline Index & SCC & P-value \\
\hline Age & 0.307 & $<0.001$ \\
BMI & 0.160 & 0.035 \\
PLT & -0.295 & $<0.001$ \\
ALT & 0.060 & 0.435 \\
AST & 0.274 & $<0.001$ \\
GGT & 0.373 & $<0.001$ \\
ALP & 0.378 & $<0.001$ \\
ALB & -0.507 & $<0.001$ \\
GLOB & 0.269 & $<0.001$ \\
A/G ratio & -0.414 & $<0.001$ \\
BILT & 0.267 & $<0.001$ \\
DBIL & 0.291 & $<0.001$ \\
IBIL & 0.222 & 0.004 \\
CHEX & -0.399 & $<0.001$ \\
PT & 0.324 & $<0.001$ \\
PTA & -0.282 & $<0.001$ \\
FIB & 0.005 & 0.946 \\
\hline SCC, & &
\end{tabular}

SCC, Spearman's correlation coefficient; BMI, body-mass index; PLT, platelet; ALT, alanine aminotransferase; AST, aspartate aminotransferase; GGT, $\gamma$-glutamyl transpeptidase; ALP, alkaline phosphatase; ALB, serum albumin; GLOB, globulin; A/G, albumin/globulin; BILT, total bilirubin; DBIL, direct bilirubin; IBIL, indirect bilirubin; CHEX, cholinesterase; PT, prothrombin time; PTA, prothrombin activity; FIB, fibrinogen.

in each patient. The results were indicated by liver stiffness measurement (LSM) in $\mathrm{kPa}$. These measurements were considered to be reliable only when the interquartile range was $<1 / 3$ of the median, and the success rate (times of successful capture of echoes / total emitting times) was $\geq 60 \%$. The detection was considered as failed in cases when the detection value was not obtained.

Statistical analysis. All the data were analyzed by SPSS version 13.0 software (SPSS, Inc., Chicago, IL, USA). The correlation between LSM and the variables was analyzed by Spearman's correlation. Multiple regression analysis was also performed to analyze the independent predictors affecting Fibroscan detection and to obtain the multiple regression equation. Differences with $\mathrm{P}<0.05$ were considered as statistically significant.

\section{Results}

Clinical features of patients. As shown in Table I, the pathological results of the 181 cases showed that patients with hepatitis B accounted for $85.1 \%$ of all included cases and patients with autoimmune diseases (including autoimmune hepatitis, primary biliary cirrhosis and primary sclerosing cholangitis) accounted for $9.9 \%$. The proportion of patients with hepatitis $\mathrm{C}(1.1 \%)$, fatty liver $(1.7 \%)$ and non-specific liver damage (2.2\%) was relatively small. The LSM value ranged between 2.5 and $75.0 \mathrm{kPa}$, with the mean value of $11.9 \pm 11.9 \mathrm{kPa}$. Biochemical indexes are also shown in Table I, including BMI, PLT, ALT, AST, GGT, ALP, ALB, GLOB, A/G, BILT, DBIL, IBIL, CHEX, PT, PTA and FIB. In the majority of patients, BMI, PLT, ALP, GLOB, ALB/GLOB ratio, BILT, DBIL, IBIL, CHEX and FIB were normal; in more than half of total patients, ALT and/or AST and/or GGT were elevated.

Correlation analysis. To determine the association between the LSM and the clinical indexes of patients, Spearman's correlation analysis was performed. As shown in Table II, the factors of age, BMI, AST, GGT, ALP, GLOB, BILT, DBIL, IBIL and PT were positively corrected with LSM $(\mathrm{P}<0.05)$. By contrast, the factors of PLT, ALB, A/G, CHEX and PTA were negatively corrected with LSM $(\mathrm{P}<0.05)$. However, there was no correlation between ALT and LSM, or between FIB and LSM. These results suggest that the factors of age, BMI, AST, GGT, ALP, GLOB, BILT, DBIL, IBIL, PT, PLT, ALB, A/G, CHEX and PTA are correlated with LSM, and that they may affect the detection results of Fibroscan.

Multiple regression analysis. To determine the independent affecting factors of Fibroscan detection, multiple regression analysis was conducted. Collinearity diagnosis among variables was performed prior to analysis. As shown in Table III, there was collinearity among ALB, GLOB and A/G, among TBIL, DBIL and IBIL, and among PT and PTA. In addition, this collinearity had a negative influence on the stability of the regression equation. Therefore, the variables of GLOB, A/G, DBIL, IBIL and PT were removed from multiple regression analysis. Multiple regression analysis was then performed on the indexes of age, BMI, PLT, AST, GGT, ALP, ALB, TBIL, PTA and CHEX. Table III demonstrates that the effects of PLT, ALB, PTA and BMI on LSM were statistically significant (all $\mathrm{P}<0.05$ ). The tolerance and variance inflation factors in the table all showed that there was no collinearity among these indicators, and the constant terms were significant. Thus, PLT, ALB, PTA and BMI were found to be independent predictors of LSM. Therefore, the regression equation was as follow: $\mathrm{Y}=47.087-0.244 \mathrm{X}_{1}-0.217 \mathrm{X}_{2}-0.205 \mathrm{X}_{3}+0.163 \mathrm{X}_{4}$, where $\mathrm{X}_{1}$ is PLT (in $x 10^{3} / \mathrm{mm}^{3}$ ), $X_{2}$ is ALB (in $\mathrm{g} / \mathrm{l}$ ), $\mathrm{X}_{3}$ is PTA (in \%) and $\mathrm{X}_{4}$ is BMI (in $\mathrm{kg} / \mathrm{m}^{2}$ ).

These results suggest that the contribution of these four predictors to the regression equation was in the following descending order: PLT (negative correlation) $>$ ALB (negative correlation) $>$ PTA (negative correlation) $>$ BMI (positive correlation).

\section{Discussion}

Fibroscan is a non-invasive method for assessing liver stiffness, which is used as an alternative method to liver biopsy. Marcellin et al (20) reported that Fibroscan was reliable for the detection of significant fibrosis or cirrhosis in HBV patients. A previous meta-analysis (21) concluded that Fibroscan theoretically has good sensitivity and specificity for cirrhosis, but it should be used with caution. In addition, Sagir et al (22) observed that the LSM value increased in patients with acute liver injury, even in cases with no evident liver fibrosis, suggesting that Fibroscan is not suitable for detecting 
Table III. Multiple regression analysis results of liver stiffness measurement influencing factors.

\begin{tabular}{|c|c|c|c|c|c|c|c|}
\hline \multirow{2}{*}{$\begin{array}{l}- \\
\text { Factor }\end{array}$} & \multicolumn{2}{|c|}{ Unstandardized coefficients } & \multirow{2}{*}{$\begin{array}{c}\begin{array}{c}\text { Standardized } \\
\text { coefficients }\end{array} \\
\text { Beta value }\end{array}$} & \multicolumn{4}{|c|}{ Collinearity diagnostics } \\
\hline & B-value & Standard error & & t-test & P-value & Tolerance & VIFs \\
\hline Constant & 47.087 & 15.420 & & 3.054 & 0.003 & & \\
\hline Age & -0.014 & 0.091 & -0.012 & -0.149 & 0.882 & 0.740 & 1.351 \\
\hline BMI & 0.542 & 0.252 & 0.163 & 2.150 & 0.033 & 0.786 & 1.271 \\
\hline PLT & -0.046 & 0.014 & -0.244 & -3.278 & 0.001 & 0.813 & 1.230 \\
\hline AST & -0.004 & 0.023 & -0.013 & -0.159 & 0.874 & 0.638 & 1.568 \\
\hline GGT & 0.022 & 0.013 & 0.178 & 1.634 & 0.105 & 0.378 & 2.647 \\
\hline ALP & 0.026 & 0.016 & 0.159 & 1.593 & 0.114 & 0.452 & 2.211 \\
\hline ALB & -0.594 & 0.281 & -0.217 & -2.113 & 0.037 & 0.427 & 2.340 \\
\hline CHEX & -0.008 & 0.015 & -0.056 & -0.553 & 0.581 & 0.432 & 2.313 \\
\hline PTA & -0.165 & 0.061 & -0.205 & -2.697 & 0.008 & 0.782 & 1.280 \\
\hline BILT & 0.026 & 0.019 & 0.109 & 1.390 & 0.167 & 0.729 & 1.371 \\
\hline
\end{tabular}

VIF, variance inflation factor; BMI, body-mass index; PLT, platelet; AST, aspartate aminotransferase; GGT, $\gamma$-glutamyl transpeptidase; ALP, alkaline phosphatase; ALB, serum albumin; CHEX, cholinesterase; PTA, prothrombin activity; BILT, total bilirubin.

cirrhosis/fibrosis in these patients. These previous findings indicated that there are various factors affecting Fibroscan detection.

In the present study, the factors influencing Fibroscan detection were analyzed. The correlation analysis demonstrated that the correlation of LSM with the indexes FIB and ALT was poor, indicating that FIB and ALT may not affect the Fibroscan detection. Subsequently, multiple regression analysis was performed in the current study. Since the results of multiple regression analysis are different if different indexes are introduced, collinearity diagnosis among the included variables was performed. A common problem in multiple regression analysis is the evident linear relation among numerous independent variables. This problem may result in an incorrect regression equation, such as an extremely unstable estimated value, sensitivity to the independent variables included in the equation, or incorrect positivity or negativity of the estimated value (23). Thus, diagnosis on multicollinearity was performed prior to estimating the multiple regression equation in the present study. According to the results, the following indexes were removed based on the collinearity diagnosis: GLOB, A/G, DBIL, IBIL and PT. Thus, the age, BMI, PLT, AST, GGT, ALP, ALB, TBIL, PTA and $\mathrm{CHEX}$ were selected for further analysis. Through multiple regression analysis, the four factors PLT, ALB, PTA and BMI were found to be independent predictors and were entered into the equation. The standardized coefficients listed in Table III showed that the contribution of these four predictors to the equation was as follows: PLT (negative correlation) $>$ ALB (negative correlation) $>$ PTA (negative correlation) $>$ BMI (positive correlation).

Although PLTs are mainly produced by the megakaryocytes of mature myeloid cells, they are also regulated by thrombopoietin produced by hepatocytes. With the evolvement of liver tissue fibrosis, thrombopoietin levels decrease, while
PLT production is reduced $(24,25)$. It is well known that the liver is the only human organ that synthesizes ALB. When the liver cells are necrotized due to various causes and are replaced by fibrous tissue, the ALB level decreases. Therefore, ALB is a sensitive index evaluating the synthetic function of liver cells and is also an important index for liver cirrhosis diagnosis. All blood coagulation factors with the exception of calcium ions and factor VIIa, are synthesized in the liver. The decrease of PTA activity and the aggravation of liver cell damage are associated with the decrease of absorbing barrier of vitamin $\mathrm{K}$ for heparin inactivation. In obese individuals, fatty liver will develop into liver fibrosis (26), and the thickness of fat layer on the abdominal wall may lead to overestimation of the LSM value (27).

The results of the present study regarding ALT and AST were inconsistent with a previous report (28). Tapper et al (28) showed that in patients with HCV infection and early-stage fibrosis, increased levels of ALT correlated with liver stiffness among patients in the lowest strata of fibrosis (METAVIR scores 0-2). The present study showed different results. In 181 patients, with 154 cases positive in HbsAg, ALT and AST had no statistically significant association with LSM. In the present study, ALT and FIB were removed from the correlation analysis, and then AST was removed from the multiple regression analysis. However, if ALT or AST were included in the regression analysis, the final calculation result would still be satisfactory, since the effect of ALT or AST on LSM is relatively small when there are more independent variables.

In conclusion, the findings of the present study indicated that when detecting the liver stiffness by Fibroscan, the parameters PLT, ALB, PTA and BMI may affect the detection results. Therefore, the diagnosis and evaluation of liver fibrosis should comprehensively consider the results of Fibroscan along with the clinical and laboratory examination results. Evaluating the 
degree of liver fibrosis simply based on the LSM value is not accurate.

\section{References}

1. Liang X, Bi S, Yang W, Wang L, Cui G, Cui F, Zhang Y, Liu J, Gong X, Chen Y, et al: Epidemiological serosurvey of hepatitis B in China-declining HBV prevalence due to hepatitis B vaccination. Vaccine 27: 6550-6557, 2009.

2. el-Serag HB: Epidemiology of hepatocellular carcinoma. Clin Liver Dis 5: 87-107, 2001.

3. Wright TL and Lau JY: Clinical aspects of hepatitis B virus infection. Lancet 342: 1340-1344, 1993.

4. Chen J, Eslick GD and Weltman M: Systematic review with meta-analysis: Clinical manifestations and management of autoimmune hepatitis in the elderly. Aliment Pharmacol Ther 39: $117-124,2014$

5. Aleman S, Rahbin N, Weiland O, Davidsdottir L, Hedenstierna M, Rose N, Verbaan H, Stål P, Carlsson T, Norrgren H, et al: A risk for hepatocellular carcinoma persists long-term after sustained virologic response in patients with hepatitis $\mathrm{C}$-associated liver cirrhosis. Clin Infect Dis 57: 230-236, 2013.

6. Angeli P, Sanyal A, Moller S, Alessandria C, Gadano A, Kim R, Sarin SK and Bernardi M; International Club of Ascites: Current limits and future challenges in the management of renal dysfunction in patients with cirrhosis: Report from the International Club of Ascites. Liver Int 33: 16-23, 2013.

7. Sharara AI, Hunt CM and Hamilton JD: Hepatitis C. Ann Intern Med 125: 658-668, 1996

8. Masuzaki R, Tateishi R, Yoshida H, Goto E, Sato T, Ohki T, Imamura J, Goto T, Kanai F, Kato N, et al: Prospective risk assessment for hepatocellular carcinoma development in patients with chronic hepatitis $\mathrm{C}$ by transient elastography. Hepatology 49: 1954-1961, 2009.

9. Castéra L, Vergniol J, Foucher J, Le Bail B, Chanteloup E, Haaser M, Darriet M, Couzigou P and De Lédinghen V: Prospective comparison of transient elastography, Fibrotest APRI, and liver biopsy for the assessment of fibrosis in chronic hepatitis C. Gastroenterology 128: 343-350, 2005.

10. Colletta C, Smirne C, Fabris C, Toniutto P, Rapetti R, Minisini R and Pirisi M: Value of two noninvasive methods to detect progression of fibrosis among HCV carriers with normal aminotransferases. Hepatology 42: 838-845, 2005.

11. Degos F, Perez P, Roche B, Mahmoudi A, Asselineau J, Voitot H and Bedossa P; FIBROSTIC study group: Diagnostic accuracy of FibroScan and comparison to liver fibrosis biomarkers in chronic viral hepatitis: A multicenter prospective study (the FIBROSTIC study). J Hepatol 53: 1013-1021, 2010

12. Sebastiani G, Halfon P, Castera L, Pol S, Thomas DL, Mangia A, Di Marco V, Pirisi M, Voiculescu M, Guido M, et al: SAFE biopsy: A validated method for large-scale staging of liver fibrosis in chronic hepatitis C. Hepatology 49: 1821-1827, 2009.

13. Bonder A and Afdhal NH: Biopsy no more; changing the screening and diagnostic algorithm for hepatitis C. Clin Gastroenterol Hepatol 11: 309-310, 2013.

14. Foucher J, Chanteloup E, Vergniol J, Castéra L, Le Bail B, Adhoute X, Bertet J, Couzigou P and de Lédinghen V: Diagnosis of cirrhosis by transient elastography (FibroScan): A prospective study. Gut 55: 403-408, 2006.
15. Nguyen-Khac E, Chatelain D, Tramier B, Decrombecque C, Robert B, Joly JP, Brevet M, Grignon P, Lion S, Le Page L and Dupas JL: Assessment of asymptomatic liver fibrosis in alcoholic patients using Fibroscan: Prospective comparison with seven non-invasive laboratory tests. Aliment Pharmacol Ther 28: 1188-1198, 2008.

16. Mueller S, Millonig G, Sarovska L, Friedrich S, Reimann FM, Pritsch M, Eisele S, Stickel F, Longerich T, Schirmacher P and Seitz HK: Increased liver stiffness in alcoholic liver disease: Differentiating fibrosis from steatohepatitis. World J Gastroenterol 16: 966-972, 2010.

17. Obara N, Ueno Y, Fukushima K, Nakagome Y, Kakazu E, Kimura O, Wakui Y, Kido O, Ninomiya M, Kogure T, et al: Transient elastography for measurement of liver stiffness measurement can detect early significant hepatic fibrosis in Japanese patients with viral and nonviral liver diseases. J Gastroenterol 43: 720-728, 2008.

18. Vergniol J, Foucher J, Mamou K, Castéra L, Bernard PH, Couzigou $\mathrm{P}$ and de Lédinghen V: Non-invasive evaluation of liver fibrosis using FibroScan in long-term sustained-virological responder patients after $\mathrm{HCV}$ treatment. Gastroenterol Clin Biol 32: 1061-1063, 2008.

19. Wang JH, Changchien CS, Hung CH, Tung WC, Kee KM, Chen $\mathrm{CH}, \mathrm{Hu} \mathrm{TH}$, Lee $\mathrm{CM}$ and Lu SN: Liver stiffness decrease after effective antiviral therapy in patients with chronic hepatitis C: Longitudinal study using FibroScan. J Gastroenterol Hepatol 25: 964-969, 2010.

20. Marcellin P, Ziol M, Bedossa P, Douvin C, Poupon R, de Lédinghen $\mathrm{V}$ and Beaugrand $\mathrm{M}$ : Non-invasive assessment of liver fibrosis by stiffiness measurement in patients with chronic hepatitis B. Liver Int 29: 242-247, 2009.

21. Tsochatzis EA, Gurosamy KS, Ntaoula S, Cholongitas E, Davidson BR and Burroughs AK: Elastography for the diagnosis of severity of fibrosis in chronic liver disease: A meta-analysis of diagnostic accuracy. J Hepatol 54: 650-659, 2011.

22. Sagir A, Erhardt A, Schmitt M and Häussinger D: Transient elastography is unreliable for detection of cirrhosis in patients with acute liver damage. Hepatology 47: 592-595, 2008.

23. Fildes R: Conditioning diagnostics: Collinearity and weak data in regression. Technometrics 35: 85-86, 1993

24. Tana MM, Zhao X, Bradshaw A, Moon MS, Page S, Turner T, Rivera E, Kleiner DE and Heller T: Factors associated with the platelet count in patients with chronic hepatitis $\mathrm{C}$. Thromb Res 135: 823-828, 2015.

25. Lu SN, Wang JH, Liu SL, Hung $\mathrm{CH}$, Chen $\mathrm{CH}$, Tung HD, Chen TM, Huang WS, Lee CM, Chen CC and Changchien CS: Thrombocytopenia as a surrogate for cirrhosis and a marker for the identification of patients at high-risk for hepatocellular carcinoma. Cancer 107: 2212-2222, 2006

26. Henderson NC and Iredalc JP: Liver fibrosis: Cellular machanisms of progression and resolution. Clin Sci (Lond) 112: 265-280, 2007.

27. Cournane S, Browne JE and Fagan AJ: The effects of fatty deposits on the accuracy of the Fibroscan ${ }^{\circledR}$ liver transient elastography ultrasound system. Phys Med Biol 57: 3901-3914, 2012.

28. Tapper EB, Cohen EB, Patel K, Bacon B, Gordon S, Lawitz E, Nelson D, Nasser IA, Challies T and Afdhal N: Levels of alanine aminotransferase confound use of transient elastography to diagnose fibrosis in patients with chronic hepatitis $\mathrm{C}$ virus infection. Clin Gastroenterol Heaptol 10: 932-937.e1, 2012. 